Meta

Journal des traducteurs

Translators' Journal

\title{
Dictionnaires et traductologie : le paradoxe d'une lointaine proximité
}

\section{Philippe Humblé}

Volume 55, numéro 2, juin 2010

URI : https://id.erudit.org/iderudit/044243ar

DOI : https://doi.org/10.7202/044243ar

Aller au sommaire du numéro

\section{Éditeur(s)}

Les Presses de l'Université de Montréal

\section{ISSN}

0026-0452 (imprimé)

1492-1421 (numérique)

Découvrir la revue

Citer cet article

Humblé, P. (2010). Dictionnaires et traductologie : le paradoxe d'une lointaine proximité. Meta, 55(2), 329-337. https://doi.org/10.7202/044243ar
Résumé de l'article

Bien que les dictionnaires soient les principaux instruments de travail des traducteurs, leur relation mutuelle n'a, jusqu'à présent, jamais été analysée en profondeur. L'usage que font les traducteurs des dictionnaires n'a guère fait l'objet de recherches, et le fait que les lexicographes bilingues sont en fin de compte des traducteurs eux-mêmes n'est jamais pris en compte. C'est cette question qui est soulevée par le présent article, lequel fournit une première ébauche de ce type de recherches. Si l'on compare les traductions de quelques phrases-exemples dans trois dictionnaires bilingues anglais-français, les problèmes rencontrés par les lexicographes ne paraissent pas tellement différents de ceux des traducteurs littéraires, ce qui constitue un terrain de recherche à explorer. 


\title{
Dictionnaires et traductologie: le paradoxe d'une lointaine proximité
}

\author{
PHILIPPE HUMBLÉ \\ Erasmushogeschool Brussel, Bruxelles, Belgique \\ philippe.humble@gmail.com
}

\begin{abstract}
RÉSUMÉ
Bien que les dictionnaires soient les principaux instruments de travail des traducteurs, leur relation mutuelle n'a, jusqu'à présent, jamais été analysée en profondeur. L'usage que font les traducteurs des dictionnaires n'a guère fait l'objet de recherches, et le fait que les lexicographes bilingues sont en fin de compte des traducteurs eux-mêmes n'est jamais pris en compte. C'est cette question qui est soulevée par le présent article, lequel fournit une première ébauche de ce type de recherches. Si l'on compare les traductions de quelques phrases-exemples dans trois dictionnaires bilingues anglais-français, les problèmes rencontrés par les lexicographes ne paraissent pas tellement différents de ceux des traducteurs littéraires, ce qui constitue un terrain de recherche à explorer.
\end{abstract}

\begin{abstract}
In spite of the fact that dictionaries are without doubt the main tools used by translators, the relationship between them earned very little scholarly attention up to now, either from lexicography scholars or from translation researchers. The fact is, nevertheless, that bilingual lexicographers are translators and are supposedly confronted with problems comparable to common translators. This article analyses briefly a set of examples from three bilingual dictionaries. The conclusion is that the problems confronted by lexicographers are, in the end, not very different from those confronted by literary translators and constitute a fascinating field of research.
\end{abstract}

\section{MOTS-CLÉS/KEYWORDS}

lexicographie, traducteurs, dictionnaires bilingues, exemples, choix lexicography, translators, bilingual dictionaries, examples, choice

\section{Introduction}

Si une enquête se proposait d'interroger les traducteurs au sujet de leurs instruments de travail, il va sans dire que le dictionnaire figurerait à la première place. D'aucuns ne manqueront de mentionner l'avènement des nouvelles technologies, à vrai dire plus tellement nouvelles, dont personne ne doutera qu'elles ont profondément bousculé la vaste recherche d'équivalences possibles entre les langues, mais elles n'ont pas remplacé le dictionnaire. Le corpus, qu'il ait été créé de toutes pièces ou qu'il soit constitué de fragments de discours, tirés pêle-mêle du gouffre "googlien", a substitué dans nombre de cas le dictionnaire pour un type de recherches auxquelles celui-ci n'était d'ailleurs pas particulièrement adapté: ce sont celles qui ont tendance à ne pas aboutir à cause de l'ordre alphabétique auquel la lexicographie s'est condamnée depuis, peu ou prou, 500 ans. Cet ordre alphabétique, évidemment, Google l'ignore.

Toutefois, en dépit de la dépendance du traducteur à l'égard du dictionnaire, l'état de la question sur les relations entre les traducteurs et la lexicographie se fait 
assez rapidement. Il existe très peu de travaux qui se soient penchés spécifiquement sur la relation qui existe entre le traducteur et le dictionnaire. Le problème n'est guère abordé en tant que tel, pas même dans des ouvrages dont l'objet est de donner une vision panoramique des récentes avancées en traductologie. Ainsi, la très complète Encyclopedia of Translation Studies de Mona Baker (1998/2004) ne compte aucun article dédié au dictionnaire, à la lexicographie ou à la lexicologie, et aucun de ces trois mots n'apparaît d'ailleurs dans l'index. On s'étonne, en outre, de ce que le mot dictionnaire ne se rencontre que rarement dans le corps du texte. Ceci en ce qui concerne les traducteurs. Qu'en est-il des lexicographes?

\section{Les lexicographes}

Du côté de la lexicographie bilingue, le bilan paraît plus mitigé. La traduction est l'occupation quotidienne des lexicographes bilingues et ils ont à tout moment conscience de leur statut de traducteurs. On serait, par conséquent, tenté de penser que le lexicographe bilingue aurait plus conscience des dangers que comporte son acte traductionnel, que le traducteur des dangers que comporte l'utilisation du dictionnaire.

Néanmoins, et à n'en pas douter, le statut de traducteur attribué au lexicographe fera sourciller plus d'un traductologue. Il ne fait pourtant pas de doute que le lexicographe bilingue, qui fait le pont entre deux langues, est un traducteur, ne serait-ce que d'une façon un peu particulière.

Avant de poursuivre notre raisonnement, il pourrait être utile de faire la distinction entre la traduction de mots (isolés) et la traduction de phrases, fait qui oppose le lexicographe au traducteur «traditionnel». Quant à la traduction de phrases, il conviendrait de séparer les séquences de mots plutôt figées de celles qui n’obéissent qu'à des contraintes grammaticales: d'un côté, nous trouvons les proverbes et les expressions idiomatiques et, de l'autre, les exemples dits «libres». Nous appelons «libres» les exemples librement composés par le lexicographe/rédacteur et, ensuite, librement traduits par le lexicographe/traducteur.

Notons que les lexicographes eux-mêmes ne sont pas d'accord sur la nature des exemples traduits dans les dictionnaires (voir Welker 2004: 149-176). Nous établissons donc nos propres critères. Dans l'article consacré à Tromper dans le Collins French-English Dictionary ${ }^{1}$, nous ne considérons pas "se tromper d'adresse», ni «tromper quelqu'un sur quelque chose» comme des exemples libres. Le premier cas est un syntagme présentant un certain degré de figement, et le second fait état de la structure sémantique du verbe. Nous considérons, par contre, comme un exemple libre une phrase telle que "il a trompé la surveillance de ses gardes et s'est enfui». Si «tromper la surveillance de» n'est assurément pas libre, cette 'surveillance' n'est pas nécessairement exercée par des 'gardes', et la personne surveillée ne s'enfuit pas nécessairement... Autrement dit, l'ensemble de la phrase n'est pas une combinaison de mots inévitable et, contrairement aux exemples précédents, il s'agit en fait d'un extrait de discours.

Les problèmes rencontrés dans la traduction de mots sont différents de ceux que l'on rencontre dans la traduction de phrases. Dans le cas de mots, il s'agit d'une problématique d'équivalence, de problèmes de couverture sémantique inégale, thèmes dont le traitement, dans les manuels de lexicographie standards, ne date pas d'hier (Zgusta 1971). Il en va de même pour la traduction de proverbes et d'autres 
expressions idiomatiques. Par contre, c'est en traduisant des phrases librement construites que le lexicographe rencontre les mêmes difficultés que celles qui sont rencontrées par le traducteur, puisque rajoutées alors aux contraintes purement lexicales celles, plus complexes, du discours.

\section{Les traducteurs}

Serait-ce parce que la relation entre le traducteur et les dictionnaires est tellement évidente que la littérature en traductologie ne semble guère lui accorder de l'importance? Dans le cas de la lexicographie, vue sous l'angle de la discipline universitaire, nous rencontrons la même situation, bien que d'une façon moins explicite. Nous n'avons repéré qu'un seul article qui traite du sujet (Rogers et Khurshid 1998) et il ne traite que de la traduction technique. De leur côté, les lexicographes semblent avoir une conscience relativement aigüe du problème: articles et chapitres qui traitent $\mathrm{du}$ problème de l'équivalence sont légion. On y trouve toutefois peu de références à la traductologie et le cadre référentiel est plutôt celui de la sémantique.

Pourtant, force est de reconnaître qu'un dictionnaire équivaut à un gigantesque effort de traduction, les langues en présence étant souvent envisagées avec un souci certain d'exhaustivité. Il est vrai qu'il ne s'agit pas là d'une traduction au sens courant du terme. En effet, la traduction, telle qu'elle est envisagée par les traductologues, est essentiellement une traduction de textes, point sur lequel il nous faudra revenir, et elle ne fait pas le départ entre traductions de mots et de phrases plus ou moins libres. Comment les lexicographes envisagent-ils donc la traduction? Un classique de la lexicographie comme Zgusta nous en donne une idée.

This lexical anisomorphism causes that the task of translating texts from one language into another is not easy; still it is possible, because the translator is not obliged to produce a word-for-word translation. To take an oversimplified example: a person in a novel is characterized by his impolite speech in a passage, this is expressed by his use of impolite words referring to, say, eating. If the target language happens to have no full equivalents (i.e. no equivalent words designating eating with the connotation of being impolite), the translator cannot but use those words of the target language which have the same designation (eating), and characterizes the speaker by the choice of some other impolite expressions. In this way, there is no absolute one-to-one correspondence between some single words of the original and those of the translation, but the whole meaning of the original passage is conveyed by its translated counterpart (Zgusta 1971: 296-297).

S’attarder à quelques présupposés exprimés dans ce passage n’est pas inutile. Bien que foncièrement exacts, les propos de Zgusta risquent de faire sourire les traductologues au fait des développements de leur discipline survenus au cours de ces vingt dernières années. Les problèmes de traduction mentionnés par Zgusta découlent essentiellement de la façon différente dont les langues découpent le monde, d'une part, et de l'expérience que nous en avons, de l'autre. Cela est un concept bien cher aux linguistes anglophones et qui, nous le savons, se rattachent à la tradition issue de Humboldt. Il s'agit d'un problème qui se résout par un procédé nommé compensation (Vinay et Darbelnet 1977/1994: 188): si une communauté linguistique n’a pas jugé nécessaire de faire une distinction donnée dans sa langue, elle en fait d'autres, d'une autre façon, et le traducteur habile saura compenser le manque. 
Il est à noter que le «subterfuge» ne peut s'appliquer qu'à la traduction de textes, et qu'il ne prend nullement en compte les problèmes de traduction tels qu'ils se manifestent dans la traduction dictionnairique. La traduction, pour Zgusta et pour les lexicographes bilingues en général, est un problème qui se manifeste au niveau des mots et qui, de ce fait, tombe sous la coupe de la polysémie. Le problème ne se situe pas au niveau du texte, qui est le domaine propre des traducteurs non-lexicographes et qui, donc, ne peut pas être résolu par des techniques inhérentes à la nature propre d'un texte. Entre les deux, le texte et les mots, rien ne semble exister. Et pourtant ce n'est pas de mots seulement que vit le dictionnaire. Une analyse plus détaillée de son contenu révèle en fait des problèmes typiques de traduction.

Tout d'abord, parmi les mots et les ensembles de mots que le dictionnaire réunit dans sa nomenclature, il y en a qui sont plus textuels que d'autres. Nous avons effectivement affaire à des mots isolés, à des expressions idiomatiques, mais aussi à des exemples. La traduction de mots isolés comme entités minimales de signification a été critiquée de façon répétée par les lexicologues, ainsi que par les sémanticiens. Le lexicographe/traducteur ne prend part à ce débat que comme consignataire. Il entend les mots et les rend dans la signification minimale qu'ils possèdent, dans le sens qu'ils recouvrent - à grand-peine - en dehors de tout contexte.

Dans le cas des expressions idiomatiques, qui sont des agencements de mots où le choix n'opère qu'au niveau d'unités supralexicales, les problèmes de traduction relèvent d'un dilemme très familier aux traducteurs, et ce, depuis toujours: celle du mot à mot ou celle du sens. Néanmoins, étant donné les objectifs pratiques du dictionnaire, le lexicographe/traducteur n'hésite pas à trancher en faveur du sens, s'opposant en cela à tous les tenants des doctrines de Schleiermacher, Benjamin et Venuti qui nous invitent à mener le lecteur vers le texte et non pas le texte vers le lecteur. Schleiermacher le dit de la façon la plus archétypique: «Entweder der Uebersezer läßt den Schriftsteller möglichst in Ruhe, und bewegt den Leser ihm entgegen; oder er läßt den Leser möglichst in Ruhe und bewegt den Schriftsteller ihm entgegen » (Ou bien le traducteur laisse l'auteur en paix et mène le lecteur vers lui, ou il laisse le lecteur en paix et lui amène l'auteur; traduit par l'auteur; Heidermann 2001 : 76). Ceci n'est pas une alternative ouverte au traducteur lexicographique.

Ainsi un exemple dans le Collins French-English Dictionary pour FINIR tel que «tu finis par m'ennuyer», est traduit par "you're beginning to annoy me». On ne fait pas de concessions, quelles qu'elles soient, pour rapprocher le lecteur d'une autre vision du monde linguistique quelconque. Ce n'est pas parce que l'on finit en français que l'on suggère d'utiliser to finish en anglais. Ainsi, à moins qu'il ne s'agisse d'expressions, les traductions dans un dictionnaire sont essentiellement axées sur le mot, et non pas sur le texte, tout simplement parce que celui-ci manque. Le problème que cela soulève est atténué tant bien que mal par la mention de synonymes, petits mondes créés par le choc des groupes de mots, ou l'ajout d'étiquettes évocatrices de paysages dans lesquels le mot à traduire pourrait s'incruster. La traduction, dans ce cas, ne paraît guère digne de l'aura que lui attribuent ses théoriciens.

Restent les exemples. La lecture d'exemples n'est décidément pas le passe-temps favori des traductologues. Les exemples dans les dictionnaires ne manquent pourtant pas d'attrait. Cela s'explique. Ce n'est qu'à partir de ces agencements que le dictionnaire acquiert valeur de texte, qu'il s'élève au-dessus du niveau de répertoire pur et 
simple. Que le lecteur considère la séquence suivante trouvée sous THING dans le Collins French-English Dictionary ${ }^{2}$ :

$\underline{\text { such things as money, fame .../des choses comme l'argent, la gloire ...; he's interested }}$ in ideas rather than things/ce qui l'intéresse ce sont les idées et non pas les objets; things of the mind appeal to him/il est attiré par les choses de l'esprit. the thing he loves most is his car/ce qu'il aime le plus au monde c'est sa voiture.

Voilà qui laisse le lecteur bien songeur! Car c'est bien de lecteur dont il s'agit ici, et non pas de simple usager, si l'on envisage l'article comme le lexicographe l'aura sans doute conçu, c'est-à-dire comme une séquence continue reflétant un enchaînement d'idées évoquées par le phénomène THING. Le collègue, lexicographe et traducteur, se verra obligé de suivre ce raisonnement, dont on voit mal comment il pourrait être autre chose que le reflet d'un consensus rendu nécessaire, si l'on ne veut pas perdre de vue l'objectif de renseigner linguistiquement l'usager. Que le lecteur nous permette une digression sur les exigences relatives aux exemples, envisagées sous l'angle de la coopération entre lexicographes. Il ne faut pas oublier que les lexicographes bilingues travaillent nécessairement (et au moins) à deux. Chacun d'entre eux n'est pas maître unique de son article, comme c'est le cas du dictionnaire monolingue. La coopération exige que celui qui invente l'exemple, ou le sélectionne, ou l'adapte à partir d'un corpus, reste dans les limites des convenances, tant morales que scientifiques. Si nous analysons la séquence d'exemples de THING cité plus haut, il semble clair que l'option - morale, éthique tout simplement - du lexicographe est en faveur des idées (ideas), et non pas des choses (things). Les «choses», concrètes ou abstraites, telles que l'argent et la gloire, acquièrent d'emblée une saveur peu recommandable si on les contraste avec des idées et que, significativement, des points de suspension font suite à leur mention... Que dire de ce qui l'intéresse ce sont les idées et non pas les objets? Cette "constatation», par ailleurs tout à fait fictive, est suivie immédiatement par son opposé, par le bon exemple, non pas linguistique, mais moral: il est attiré par les choses de l'esprit. Mais voilà que la bête rebondit: ce qu'il aime le plus au monde c'est sa voiture. Pour se rendre compte des contraintes extralinguistiques qui guident nécessairement le lexicographe en tant qu'inventeur d'exemples, il n'y a qu'à inverser ces exemples. Comment le partenaire du lexicographe/inventeur pourrait-il traduire une phrase telle que ce qui l'intéresse ce sont les objets et non pas les idées? Ou, pire encore, ce qu'il aime le plus au monde ce sont les idées? Voilà ce qui serait louable du point de vue des valeurs, mais hautement improbable en tant qu'énoncé de la vie quotidienne. Pour que le partenaire lexicographe/traducteur puisse traduire les exemples, le lexicographe/inventeur doit s'en tenir à des lieux communs, des banalités, mais qui acquièrent le statut de «texte». Ce qui vient avant l'exemple et ce qui le suit est donné par notre expérience personnelle, pas tellement personnelle, du monde, et que le lexicographe/traducteur se doit de respecter. Même si tel n'est pas l'objet du dictionnaire, il est quand même le dépôt de notre conception spontanée du monde à une certaine époque, ce sur quoi nous nous devons d'être tous d'accord. L'exemple, du moins celui que nous avons sélectionné et qualifié de «libre», est un texte. Un texte banal, mais un texte et non pas un agencement neutre de mots.

Une dernière remarque à ce sujet. Il est important de ne pas laisser passer inaperçu l'aspect fictionnel de tout exemple. Impossible de ne pas se figurer de situation irrépressiblement concrète à la lecture de il est attiré par les choses de l'esprit, ou ce 
qu'il aime le plus au monde c'est sa voiture. Situation vite repérée, aisée à imaginer, mais fictive de toute façon, et différente selon tout un chacun. Il s'agit d'une situation fictive imaginée par l'auteur de l'exemple, d'une autre pour le traducteur, d'une autre encore pour l'usager du dictionnaire. Plus court est l'exemple, plus il prêtera à l'envol de l'imagination. Quelle est l'information en doublure émanant de l'article? Le dictionnaire mérite une attention plus soutenue de nos collègues traductologues.

\section{Le texte}

Qu'est-ce qu'un texte? L'exemple est-il un texte? Certainement pas dans un sens traditionnel, irréfléchi. La longueur joue-t-elle un rôle? Un exemple est rarement plus long qu'une phrase. Rarement l'exemple contient-il plus d'une poignée de mots, le tout juste nécessaire pour éclairer l'utilisateur du dictionnaire sur son sens, ou sur son possible entourage lexical.

L'exemple du dictionnaire monolingue est généreux: il distrait son lecteur, il s'adresse aux connaisseurs. Le cédérom du Petit Robert ${ }^{2}$ permet de se délecter de son auteur préféré. Un clic de souris et nous voilà en présence d'une série de sources sélectes au service de la signification d'un vocable quelconque. La compagnie est privilégiée, voilà Pérec et Martin du Gard pour nous faire la leçon sur DÉLESTER, Gide et Proust pour nous guider dans l'usage de DINGUER. L'autre sens est possible également: une recherche sur Queneau débouche sur quelques centaines de phrases qui illustrent non seulement GNON et GAMBERGER, mais qui nous permet de savourer Queneau en passant. Plaisir exquis, réservé aux natifs questionnant leurs esprits parents. Mais que penser des exemples dans un dictionnaire bilingue? Ils sont d'une tout autre nature, appelés non pas à claironner la gloire d'une culture, le goût d'une civilisation, mais à rassurer le non-initié, l'apprenant, le guider, en fait l'induire en erreur, le confondre sur la complexité de la langue en apprentissage. Comparons donc les exemples pour tromper de l'Oxford-Hachette Superlex $x^{3}$ et une poignée d'exemples du Petit Robert:

(1) Nous avons été trompés par les bons résultats/la ressemblance.

On nous a trompés sur la qualité des produits/l'état de la maison.

Il y a des signes ou gestes qui ne trompent pas.

Je me suis trompé sur leurs intentions.

(Oxford-Hachette Superlex 1996)

(2) Car c'est double plaisir de tromper le trompeur. (La Fontaine)

L'art de plaire est l'art de tromper. (Vauvenargues)

Une femme qu'on aime suffit rarement à tous nos besoins et on la trompe avec une femme qu'on n'aime pas. (Proust)

(Robert 2001)

La comparaison est odieuse, mais le contraste n'en est que renforcé. Un lexicographe chargé de traduire les exemples du Petit Robert serait un vrai traducteur. Celui, bien plus réel pourtant, de l'Oxford-Hachette Superlex en sera réduit au statut de simple connaisseur de la langue, simple Dolmetscher, tolmács, Mittelsmann, entremetteur anonyme. Où donc tracer la limite entre celui-ci et le lexicographe/traducteur? Les traductologues lui nieraient-ils le statut de traducteur? Il faut d'abord déterminer ce qu'est un traducteur dans le contexte qui nous occupe ici et en quoi il se distingue de celui qui n'est pas interprète pur et simple. 


\section{L'exemple, le traducteur et les choix}

Si nous comparons les exemples que l'Oxford-Hachette Superlex, le Grand Dictionnaire Français-Anglais de Larousse ${ }^{4}$ et le Collins French-English Dictionary nous offrent pour l'article Juste (adjectif), il est manifeste qu'ils ne sont pas tous égaux.

(1) Un poulet pour six c'est un peu juste.

One chicken for six people is stretching it a bit.

(Oxford-Hachette 1996)

(2) Trois bouteilles pour sept personnes, c'est un peu juste!

Three bottles for seven people, that's cutting it rather fine.

(Larousse 1996)

(3) $1 \mathrm{~kg}$ pour 6 - c'est un peu juste.

$1 \mathrm{~kg}$ for 6 people - it's barely enough ou it's a bit on the short ou skimpy side.

(Collins French-English Dictionary 1996)

Voilà trois exemples très ressemblants, presque identiques, tellement simples en apparence qu'ils se confondent. C'est le lexicographe qui abandonne toute velléité de traducteur, adossé au mur de l'évidence. Du moins à première vue. Dans l'OxfordHachette Superlex, c'est un poulet qui est un peu juste pour six. Dans le Larousse, c'est trois bouteilles desquelles sept personnes auront très vite raison. Finalement, dans le Collins French-English Dictionary il s'agit d'un kilo d'une nourriture quelconque. La phrase coïncide: c'est un peu juste, peu importe la pitance. Pourtant, les traductions sont différentes. Le lexicographe/traducteur, qui n’avait pas le choix en traduisant la plus grande partie de l'article, s'en donne à cœur joie quand l'occasion s'en offre. Google n'est pas infaillible, bien sûr, mais reste qu'il est intéressant de comparer le nombre d'occurrences de chacune de ces locutions pour avoir une idée de leur fréquence, quitte à y trouver la raison de tant de divergences dans les convictions des lexicographes/traducteurs.

TABLEAU 1

Fréquences des traductions de c'est un peu juste, obtenues à l'aide de Google

\begin{tabular}{|l|l|c|}
\hline Dictionnaire & Traduction & $\begin{array}{l}\text { Google } \\
\mathbf{2 1} \text { mars 2010 }\end{array}$ \\
\hline Oxford-Hachette Superlex & stretching it a bit & 752000 \\
\hline Larousse & cutting it rather fine & 27300 \\
\hline Collins French-English Dictionary & barely enough & 607000 \\
\hline & a bit on the short side & 1970000 \\
\hline & a bit on the skimpy side & 60300 \\
\hline
\end{tabular}

Le lexicographe/traducteur de Collins French-English Dictionary est le plus indécis, et nous lui en savons gré. Il nous offre trois possibilités. Elles sont de fréquence variable et l'utilisateur du dictionnaire devra user de bon sens (et de Google, si les fréquences l'intéressent) pour faire son choix. Le lexicographe Collins a certainement eu assez de flair pour choisir la solution apparemment la plus populaire. Le traducteur à l'œuvre dans l'Oxford-Hachette Superlex, lui aussi, a résolument choisi de mener le lecteur parmi les sentiers sûrs de la relative popularité: 752000 occurrences. Le lexicographe du Larousse, par contre, a préféré le singulier, presque "posh» avec 
'seulement' 27300 occurrences, sans craindre que son utilisateur se distingue innocemment de ses interlocuteurs par un langage châtié outre mesure. Ou alors a-t-il donné libre cours à ses propres préférences d'expression, peu convaincu par les façons de parler en vogue? Ou peut-être s'est-il tout simplement trompé, et s'agit-il d'une erreur d'appréciation? Toujours est-il que les lexicographes ont ici pu, et dû, choisir. Ils ont usé de leur jugement, de leur expérience, de leurs convictions, de celles des autres, pour traduire un authentique fragment de texte.

Quel est le problème central de la traduction? N’est-ce pas le choix, l'inépuisable incertitude quant à l'adéquation du choix? Le lexicographe, qui s'esquive quand il s'agit de s'affirmer comme traducteur, n'en est pas moins un, et plus qu'il ne le pense. Son métier n'est pas caractérisé par cette objectivité 'indéniable', que le profane a coutume de lui attribuer. Il est mené lui aussi par des motifs personnels, corporatifs (dictés par sa maison d'édition), ou qui sont le résultat de ses convictions sur la pédagogie des langues étrangères.

Qu'y a-t-il de particulier dans ces exemples si ce n'est que ce sont des opinions sur ce qui serait de mise dans une situation que le lexicographe/traducteur s'est efforcé de s'imaginer, de rendre en fiction? L'imagination est d'une importance non négligeable dans le métier du traducteur littéraire, et le dictionnaire est, à sa façon, une œuvre littéraire. Elle demande même davantage à l'imagination de son lecteur puisque l'œuvre est essentiellement en construction. Trois bouteilles pour sept personnes, c'est un peu juste! oblige le lexicographe/traducteur et son lecteur à prescrire à l'imagination une situation, à dresser un constat sur de piètres fragments qui ne permettent d'engager que, pour l'un, le travail de traduction et, pour l'autre, sa compréhension. Il s'agit là d'une péniche bien étroite pour un canal bien houleux. Le résultat de cette opération risquera d'être toujours sujette à caution, et son succès, douteux.

\section{Conclusion}

À quelle conclusion en arrive-t-on? Au moins à la constatation que les traductologues ont grand tort de ne pas s'occuper des dictionnaires. Ceux-ci devraient être soumis à leur scrutation critique et systématique, ne serait-ce que pour de raisons sociologiques. Les dictionnaires représentent un corpus de textes pour le moins singulier, dans leur banalité nécessairement recherchée, dans leur consensus absolument requis. Est-il possible de détecter les routines des traducteurs traditionnels dans les traductions dictionnairiques? Cela a-t-il une influence sur leur efficacité? Quelle est la conscience de traducteur des lexicographes? Pourquoi, apparemment, les traducteurs/lexicographes ne se considèrent-ils pas comme tels? Les problèmes auxquels ils sont confrontés sont pourtant semblables à ceux des traducteurs littéraires. Les exemples remplissent certaines des conditions exigées pour être considérés texte. Les considérer comme tels ne contribuerait-il pas à leur perfectionnement? Il y a là tout un filon qui demande à être exploré.

\section{REMERCIEMENTS}

Je tiens à remercier la CAPES brésilienne pour la bourse d'une année sabbatique qui a rendu possible la recherche faite pour cet article; agradeço o apoio da CAPES que, por meio de uma bolsa de pósdoutorado, possibilitou a pesquisa feita para este artigo. Je tiens également à remercier le comité de rédaction de Meta pour ses commentaires constructifs. 


\section{NOTES}

1. Collins French-English Dictionary (1996): Londres: HarperCollins. Cédérom.

2. Nouveau Petit Robert 2.0 (2001) Paris: Dictionnaires Le Robert. Cédérom.

3. Oxford-Hachette Superlex (1996): Oxford University Press - Hachette livre. Cédérom.

4. Grand Dictionnaire Français-Anglais - Larousse (1996): Paris: Larousse. Cédérom.

\section{RÉFÉRENCES}

BAKer, Mona (1998/2004): Routledge Encyclopedia of Translation Studies. Routledge: London. Benjamin, Walter (1923/2001): Die Aufgabe des Übersetzers. In: Werner Heidermann, dir. Clássicos da teoria da tradução. Florianópolis: Universidade Federal de Santa Catarina, 188-215.

Drysdale, Patrick D. (1987): The role of examples in a learner's dictionary. In: Anthony CowiE, dir. The Dictionary and the Language Learner, Papers from the EURALEX Seminar at the University of Leeds. (EURALEX Seminar, Leeds, 1-3 Avril 1985) Tübingen: Niemeyer, 213-223.

Rogers Margaret and Khurshid Ahmad (1998): The translator and the Dictionary: Beyond Words? In: Atkins Beryl dir. Using Dictionaries. Studies of Dictionary Use by Language Learners and Translators. Tübingen: Niemeyer.

Schleiermacher, Friedrich (1838/2001) Ueber die verschiedenen Methoden des Uebersezens In: Werner Heidermann, dir. Clássicos da teoria da tradução. Florianópolis: Universidade Federal de Santa Catarina, 26-87.

Vinay, Jean Pierre et DARbeLnet, Jean (1977/1958): Stylistique comparée du français et de l'anglais. Paris: Didier.

Welker, Herbert A. (2004): Dicionários: Uma pequena introdução à lexicografia. Brasília: Thesaurus.

Williams, Jenny et Chesterman, Andrew (2002): The Map: A Beginner's Guide to Doing Research in Translation Studies. Manchester: St. Jerome.

Zgusta, Ladislav (1971): Manual of Lexicography. La Haye: Mouton. 